\title{
Information Indivisibility Results
}

\author{
Jossy Sayir*, Ingmar Land ${ }^{\dagger \ddagger}$, and Alex Grant ${ }^{\dagger}$ \\ Emails: sayir@ftw.at, \{ingmar.land,alex.grant $\} @$ unisa.edu.au \\ * Forschungszentrum Telekommunikation Wien (ftw.), \\ Donau-City-Str. 1, A1220 Vienna, Austria \\ $\dagger$ Institute for Telecommunications Research, \\ University of South Australia, Mawson Lakes, South Australia \\ $\ddagger$ Department of Electronic Systems, \\ Aalborg University, Denmark
}

\begin{abstract}
We derive a theorem stating that it is not possible to generate two independent observations of a binary random variable such that each observation gives only partial information about the binary variable, but the two observations jointly determine the binary variable exactly. We illustrate this theorem with examples and elaborate on applications of the theorem to cryptography and to determining the EXIT function of a zeroerror code.
\end{abstract}

\section{INTRODUCTION}

The following cryptographic scenario motivates the results presented in this paper. Despite never having met in person and always communicating through secret channels, Alice and Bob eventually became good friends and, together, discovered the answer to the fundamental question of information theory, cryptography, and everything. The exact nature of this question is outside the scope of this paper, but for the sake of the story let us assume that its answer is simply 0 or 1 . Alice and Bob agreed to keep this answer secret and never to divulge it to anyone during their lifetime. On their deathbeds, Alice and Bob gave their disciples Alfred and Beatrix a one thousand bit clue each. Each clue gives partial information about the precious answer they had discovered, but the exact answer can only be reconstituted by combining the two clues. Note that Alice and Bob lived at opposite ends of the globe, had disabled any secret channels between them at this stage and thus could not collaborate in any way to generate the two clues, nor had they had the foresight of generating common randomness in advance to help in the process. The present paper claims a fundamental flaw in Alice and Bob's plan: according to the results that will be presented, re-constituting the exact answer based on the combined clues is a mathematical impossibility, unless the exact answer is deducible from at least one of the two clues individually.

In Section II, we will introduce the main result of the paper, a theorem stating that it is not possible to generate independent observations of a binary random variable that each give only partial information but together essentially determine the variable. Section III will provide a proof of this result. In Section IV, we will show examples and discuss applications of the result, in particular its use for determining part of the EXIT curve of a zero-error channel code.

\section{The Bit Indivisibility TheOREM}

Let the random variable $X$ carry the binary secret and $Y_{1}$ and $Y_{2}$ be the two clues that grant access to the secret. By requiring that $Y_{1}$ and $Y_{2}$ be generated independently, we mean that the two variables give no information about each other when $X$ is known, or in other words that the conditional mutual information $I\left(Y_{1} ; Y_{2} \mid X\right)$ be zero. This is equivalent to the condition that $Y_{1}$ and $Y_{2}$ be independent given $X$, or that $Y_{1}-X-Y_{2}$ form a Markov chain. Generating two independent clues that jointly grant access to a binary secret is an instance of the general case treated in the following theorem:

Theorem 1 (Bit Indivisibility Theorem): Let the random variables $Y_{1}-X-Y_{2}$ form a Markov chain, where $X$ is defined over the alphabet $\{0,1\}$. Then

$$
H\left(X \mid Y_{1} Y_{2}\right)=0 \Longleftrightarrow H\left(X \mid Y_{1}\right)=0 \text { OR } H\left(X \mid Y_{2}\right)=0 .
$$

In other words, it is not possible to divide the information about a binary random variable $X$ into two independent observations $Y_{1}$ and $Y_{2}$, such that each observation gives only partial information about $X$ but the two observations jointly give full information about $X$.

Note that although the theorem is stated for two clues/observations $Y_{1}$ and $Y_{2}$, either of these could be a vector, so the theorem can be applied recursively to tackle any collection of independent observations $Y_{1}, Y_{2}, Y_{3}, \ldots$

In the next section, we will provide a proof of this result, then show examples and applications in the following section.

\section{PROOF OF THE BIT INDIVISIBILITY THEOREM}

The " $\Leftarrow$ " of Theorem 1 can be proved using the chain rule of entropies,

$$
H\left(X \mid Y_{1} Y_{2}\right) \leq H\left(X \mid Y_{1}\right) \text { and } H\left(X \mid Y_{1} Y_{2}\right) \leq H\left(X \mid Y_{2}\right),
$$

and therefore $H\left(X \mid Y_{1} Y_{2}\right)=0$ if either $H\left(X \mid Y_{1}\right)=0$ or $H\left(X \mid Y_{2}\right)=0$. The proof of the " $\Rightarrow$ " is considerably longer and will occupy the rest of this section.

Let $X, Y_{1}, Y_{2}$ be random variables as specified by the theorem. We have

$$
H\left(X \mid Y_{1} Y_{2}\right)=\sum_{y_{1} y_{2}} P\left(y_{1} y_{2}\right) H\left(X \mid Y_{1} Y_{2}=y_{1} y_{2}\right)
$$


so that $H\left(X \mid Y_{1} Y_{2}\right)$ can be zero only if $H\left(X \mid Y_{1} Y_{2}=y_{1} y_{2}\right)=$ 0 for all $y_{1}, y_{2}$ such that $P\left(y_{1} y_{2}\right)>0$. Note that we can write $P\left(y_{1} y_{2}\right)=P\left(y_{1}\right) P\left(y_{2} \mid y_{1}\right)=P\left(y_{2}\right) P\left(y_{1} \mid y_{2}\right)$ so that $P\left(y_{1} y_{2}\right)>0$ implies $P\left(y_{1}\right)>0$ and $P\left(y_{2}\right)>0$.

Using $h($.$) to denote the binary entropy function, we can$ now proceed as described in Equations 4 to 7 below, where we have used the Markov chain property in the last step. In order for $H\left(X \mid Y_{1} Y_{2}=y_{1} y_{2}\right)$ to be zero, the argument of $h($. must be either zero or one, which is only possible if

$$
P(X=0) P\left(y_{1} \mid X=0\right) P\left(y_{2} \mid X=0\right)=0
$$

or if

$$
P(X=1) P\left(y_{1} \mid X=1\right) P\left(y_{2} \mid X=1\right)=0 .
$$

If $P(X=0)=0$ or $P(X=1)=0$, then $H(X)=0$, which implies that $H\left(X \mid Y_{1} Y_{2}\right), H\left(X \mid Y_{1}\right)$ and $H\left(X \mid Y_{2}\right)$ are all zero, and the theorem holds. Otherwise, we see that either $P\left(y_{1} \mid X=0\right), P\left(y_{2} \mid X=0\right), P\left(y_{1} \mid X=1\right)$, or $P\left(y_{2} \mid X=1\right)$ must be zero. Without loss of generality, let us assume that the first of these probabilities is zero. Then,

$$
\begin{gathered}
H\left(X \mid Y_{1}=y_{1}\right)=h\left(P\left(X=0 \mid y_{1}\right)\right) \\
=h\left(\frac{P(X=0) P\left(y_{1} \mid X=0\right)}{P(X=0) P\left(y_{1} \mid X=0\right)+P(X=1) P\left(y_{1} \mid X=1\right)}\right) \\
=0 .
\end{gathered}
$$

The same applies to the other three probabilities, so we conclude that $H\left(X \mid Y_{1} Y_{2}=y_{1} y_{2}\right)=0$ if and only if $H(X)=0, H\left(X \mid Y_{1}=y_{1}\right)=0$ or $H\left(X \mid Y_{2}=y_{2}\right)=0$.

We already showed that the theorem holds if $H(X)=0$. If $H\left(X \mid Y_{1}=y_{1}\right)=0$ for all $y_{1}$ such that $P\left(y_{1}\right)>0$, then $H\left(X \mid Y_{1}\right)=0$ and the theorem holds as well. The same is true if $H\left(X \mid Y_{2}=y_{2}\right)=0$ for all $y_{2}$ such that $P\left(y_{2}\right)>0$. On the other hand, if there exists a $y_{1}$ such that $H\left(X \mid Y_{1}=y_{1}\right)>0$ and a $y_{2}$ such that $H\left(X \mid Y_{2}=y_{2}\right)>0$, then $H\left(X \mid Y_{1} Y_{2}=\right.$ $\left.y_{1} y_{2}\right)>0$. In other words, we cannot zero $H\left(X \mid Y_{1} Y_{2}\right)$ by setting $H\left(X \mid Y_{1}=y_{1}\right)=0$ for some $y_{1}$ and setting $H\left(X \mid Y_{2}=\right.$ $\left.y_{2}\right)=0$ for some $y_{2}$.

We show an example that illustrates the cases considered at the end of the proof. Let $X, Y_{1}$ and $Y_{2}$ be connected through two $Z$-channels as illustrated in Figure 1. In this case, $H\left(X \mid Y_{1}=1\right)=H\left(X \mid Y_{2}=0\right)=0$ but $H\left(X \mid Y_{1}=\right.$ $0)>0$ and $H\left(X \mid Y_{2}=1\right)>0$. We have $P_{Y_{1} Y_{2}}(1,0)=0$. But $P_{Y_{1} Y_{2}}(0,1) \neq 0$ and $H\left(X \mid Y_{1} Y_{2}\right)$ can only be zero if $H\left(X \mid Y_{1} Y_{2}=01\right)=0$, which in turn implies that one of the diagonal connections of the $Z$-channels must have probability
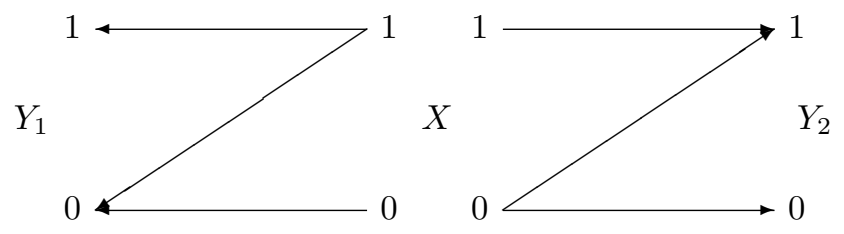

Fig. 1. Two parallel $Z$-channels

zero, effectively turning it into a noiseless channel as stipulated by the theorem. Interestingly, the joint channel from $X$ to

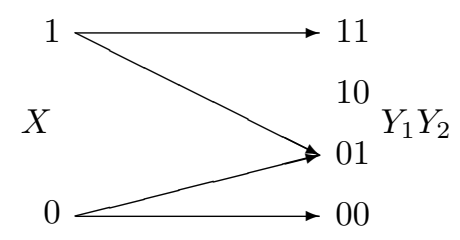

Fig. 2. Equivalent channel for two parallel $Z$ channels

$Y_{1} Y_{2}$ can be seen as an erasure channel, which is symmetric if the probabilities on the diagonal links of the $Z$-channels are equal, and noiseless if and only if the probability of an erasure given at least one of the input symbols is zero, as illustrated in Figure 2.

\section{DISCUSSION, EXAMPLES AND APPLICATIONS}

If the Markov condition is lifted, it is easy to see that there is a trivial solution to Alice and Bob's problem: let $Y_{1}$ be a uniformly distributed binary random variable independent of $X$ and let $Y_{2}=X+Y_{1}$ where the addition is taken modulo 2, i.e., in $\operatorname{GF}(2)$. Then we have $H\left(X \mid Y_{1}\right)=H\left(X \mid Y_{2}\right)=1$ but $H\left(X \mid Y_{1} Y_{2}\right)=0$, i.e., the two one-bit clues $Y_{1}$ and $Y_{2}$ give no information at all about $X$ individually, but together they determine $X$ exactly. However, Bob needs to know the $Y_{1}$ generated by Alice in order to generate the corresponding $Y_{2}$, so the two keys are not generated independently in this scenario.

A simple example also shows that the theorem does not apply to non-binary $X$ in its current form. Consider the case of a uniformly distributed quaternary random variable $X$. The four possible values of $X$ can be written as two binary digits. Let $Y_{1}$ be the first of these digits, transmitted through a

$$
\begin{aligned}
H\left(X \mid Y_{1} Y_{2}=y_{1} y_{2}\right) & =h\left(P\left(X=0 \mid y_{1} y_{2}\right)\right) \\
& =h\left(\frac{P\left(X=0, y_{1}, y_{2}\right)}{P\left(y_{1} y_{2}\right)}\right) \\
& =h\left(\frac{P(X=0) P\left(y_{1} \mid X=0\right) P\left(y_{2} \mid y_{1}, X=0\right)}{\sum_{x} P\left(x y_{1} y_{2}\right)}\right) \\
& =h\left(\frac{P(X=0) P\left(y_{1} \mid X=0\right) P\left(y_{2} \mid X=0\right)}{P(X=0) P\left(y_{1} \mid X=0\right) P\left(y_{2} \mid X=0\right)+P(X=1) P\left(y_{1} \mid X=1\right) P\left(y_{2} \mid X=1\right)}\right)
\end{aligned}
$$




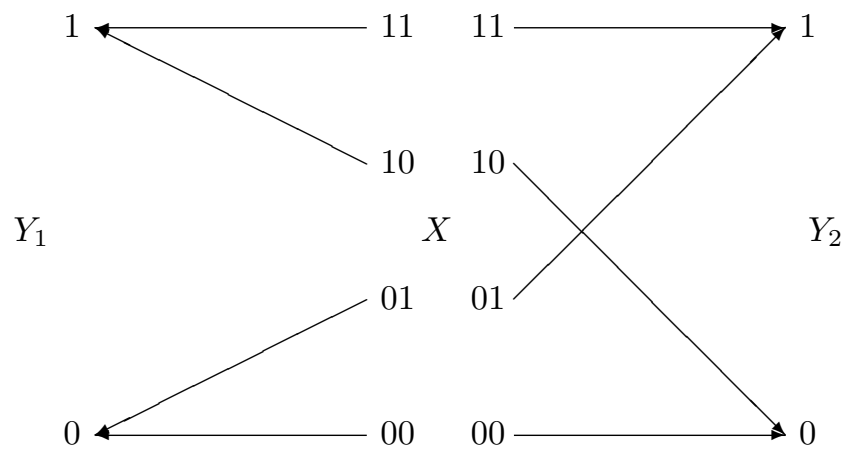

Fig. 3. Quaternary divisibility scenario

deterministic noiseless channel, and $Y_{2}$ be the second digit, as illustrated in Figure 3. Then we have

$$
I\left(Y_{1} ; Y_{2} \mid X\right)=H\left(Y_{1} \mid X\right)-H\left(Y_{1} \mid X Y_{2}\right)=0-0=0,
$$

so $Y_{1}-X-Y_{2}$ form a Markov chain as required by the theorem. However, $H\left(X \mid Y_{1}\right)=1$ bit, $H\left(X \mid Y_{2}\right)=1$ bit, while $H\left(X \mid Y_{1} Y_{2}\right)=0$, which shows that an equivalent theorem would not hold for this non-binary random variable $X$.

Beyond the anecdotal application to cryptography described in the introduction, the theorem has consequences for a variety of disciplines. In physics, the theorem implies that when measuring a binary quantity (e.g., the spin of an electron), repeated independent measurements can only determine the quantity of interest beyond doubt if one of the measurements does so on its own. In decision theory, the theorem implies that a binary decision (e.g., whether to "buy" or to "sell" a financial product on a stock exchange) based on a collection of independent criteria can only be unambiguous if one of the criteria determines the decision without ambiguity.

A further telecommunications-related application of the theorem is to determine the EXtrinsic Information Transfer (EXIT) function [1] of a zero-error code. "Zero-error" here refers to the ability of the code to provide a vanishing probability of error for channels whose capacity lies above a certain threshold. This can be interpreted in the asymptotic regime, in which case we are considering the limiting case of a "good code", or a "capacity-achieving" family of codes. EXIT charts are normally used for code design, where the EXIT functions of code components (check nodes and variable nodes for LDPC codes, or component convolutional codes for turbo codes) are designed to optimize the performance of the code. If a zero-error code is to be used itself as a component within an iterative setup, for example within a turbo equalization scheme, then the overall EXIT function of the code becomes relevant. There have been claims (e.g., [2]) that this EXIT function is a step function as indicated in Figure IV, whose value remains zero up to an a-priori mutual information corresponding to the threshold/capacity of the code, and becomes 1 beyond that threshold. While it is not trivial to prove that the mutual information is zero below
Fig. 4. Claimed EXIT function of a capacity-achieving code

the threshold, we can easily show that the mutual information is 1 above the threshold using the bit indivisibility theorem.

Let $X_{1}, X_{2}, \ldots$ be the sequence of code digits and $Y_{1}, Y_{2}, \ldots$ be the sequence of associated channel output observations, where the channel is assumed to be memoryless. We write $Y_{\backslash i}$ for the sequence of all but the $i$-th observation. Due to the memoryless nature of the channel, $Y_{\backslash i}-X_{i}-Y_{i}$ forms a Markov chain. We assume a binary code, so $X_{i}$ is a binary random variable. Since the code is error-free when we are operating above the threshold, we know that $I\left(X_{i} ; Y_{1} Y_{2} \ldots\right)=1$, or in other words $H\left(X_{i} \mid Y_{1} Y_{2} \ldots\right)=0$, i.e., the sequence of observations essentially determines every code digit. Therefore, the conditions of the theorem apply, and unless the channel is noiseless, we know that $H\left(X_{i} \mid Y_{i}\right) \neq 0$, which in turn implies that $H\left(X_{i} \mid Y_{\backslash i}\right)=0$ and

$$
I\left(X_{i} ; Y_{\backslash i}\right)=1,
$$

which is the extrinsic mutual information plotted in the EXIT function above the threshold.

\section{ACKNOWLEDGMENT}

Part of this work was performed during a visit sponsored by the European Network of Excellence NEWCOM and by the Australian Research Council's (ARC) research network ACoRN (RN0459498). The work was also supported by the ARC Discovery Grant DP0663567 and by NEWCOM++. ftw. is a research center within the Austrian goverment's COMET funding scheme.

\section{REFERENCES}

[1] S. ten Brink, Convergence behavior of iteratively decoded parallel concatenated codes, IEEE Trans. Commun., vol. 49, no. 10, pp. 17271737, Oct. 2001.

[2] M. Peleg, A. Sanderovich and S. Shamai (Shitz), On Extrinsic Information of Good Binary Codes Operating on Gaussian Channels, European Trans. on Telecom. (ETT), Vol. 18, No. 2, pp. 133-139, 2007. 